\title{
New X-Ray Tomography Method Based on the 3D Radon Transform Compatible with Anisotropic Sources
}

\author{
M. Vassholz, B. Koberstein-Schwarz, A. Ruhlandt, M. Krenkel, and T. Salditt" \\ Institut für Röntgenphysik, Georg-August-Universität Göttingen, Friedrich-Hund-Platz 1, 37077 Göttingen, Germany \\ (Received 8 September 2015; revised manuscript received 16 November 2015; published 24 February 2016)
}

In this work, we propose a novel computed tomography (CT) approach for three-dimensional (3D) object reconstruction, based on a generalized tomographic geometry with two-dimensional angular sampling (two angular degrees of freedom). The reconstruction is based on the 3D Radon transform and is compatible with anisotropic beam conditions. This allows isotropic 3D imaging with a source, which can be extended along one direction for increased flux, while high resolution is achieved by a small source size only in the orthogonal direction. This novel scheme for analytical CT is demonstrated by numerical simulations and proof-of-concept experiments. In this way high resolution and coherence along a single direction determines the reconstruction quality of the entire $3 \mathrm{D}$ data set, opening up, for example, new opportunities to achieve nanoscale resolution and/or phase contrast with low brilliance sources such as laboratory x-ray or neutron sources.

Hard x-ray tomography [1-5] offers a unique capability to nondestructively map out the three-dimensional (3D) structure within the interior of a body or material, owing to a unique penetration power and a quantitatively accountable contrast formation. Complementing the absorption contrast, which has prevailed the first hundred years of $\mathrm{x}$-ray imaging, the recent two decades have brought phase contrast $\mathrm{x}$-ray imaging to reality, enabling the 3D visualization of non- or weakly absorbing soft tissues as well as nanostructures in materials, based on advanced phase retrieval techniques [6-9]. A persistent challenge for tomography applications with high resolution and/or phase contrast is the required brilliance of the radiation source, impeding applications to be carried out with readily available laboratory sources. For neutrons, even large scale facilities like reactors or spallation sources do not provide sufficient brilliance for many high resolution or high coherence computed tomography (CT) applications, in particular, at the nanoscale.

Here we show that by suitable generalization of the tomographic measurement geometry as well as the corresponding framework of object reconstruction, some of the present limitations can be lifted. In particular, one can significantly relax the brilliance or source size in one of the two source dimensions. To this end, we replace the conventional framework of the two-dimensional Radon transform (2DRT), which to date is the common basis for analytical X-ray CT, including micro- and nano-CT, with the three-dimensional Radon transform (3DRT). We show that by proper extension of the data recording scheme, at least within geometric approximations suitable for analytical CT,

Published by the American Physical Society under the terms of the Creative Commons Attribution 3.0 License. Further distribution of this work must maintain attribution to the author(s) and the published article's title, journal citation, and DOI. the area integrals required for the 3DRT can be realized experimentally. Thereby, the measurement and reconstruction are made compatible with source properties which can be relaxed along one dimension (the "low-resolution direction"), while the beam properties along the "high-resolution direction" determine the resolution and contrast of the entire 3D object reconstruction. While several other advanced tomography concepts such as cone beam algorithms also reconstruct directly into 3D volumes [10], the present scheme is distinctly different in the angular sampling and reconstruction approach, which makes it uniquely compatible with anisotropies in the setup and offers unique advantages for local tomography. Because of the general nature of the approach, the anisotropy can concern many different beam properties (collimation, focus size, spatial coherence), the detection scheme (resolution, scanning), as well as displacements of object or optics, which are required by phase sensitive methods such as grating interferometry [8] or edge illumination [11]. This paves the way towards a new quality of micro- and nano-CT at compact and broadly accessible sources, as of other brilliance-limited tomographic probes (neutrons, electrons, ions).

To consider the starting point of this idea, we recall that the line integrals on which conventional CT is based, typically require the beam to be collimated in two directions. More precisely, the distribution function of wave vectors $p\left(n_{k}\right)$ has to be a narrow function, i.e., ideally a $\delta$ distribution. In practice, the width of this distribution has to be smaller than a critical value, which is a function of the desired resolution $\sigma_{i} \leq \sigma_{c}$, as determined by source size, detector pixel, geometry, etc. Typically, x-ray optics are used to provide this collimation, however, at the expense of flux. In contrast to synchrotron radiation (SR), which is intrinsically collimated by the superrelativistic radiation cone, the collimation at laboratory sources is compromised by a dramatic loss in flux. For this reason, the collimation 
of the beam in diffraction experiments is often relaxed in the direction orthogonal to the scattering plane, while it is collimated to the desired value in the scattering plane. By this strategy of anisotropic beam preparation, many classical diffraction experiments are enabled at laboratory sources which would otherwise be only possible with SR. Contrarily, $\mathrm{x}$-ray imaging and tomography involve a $2 \mathrm{D}$ projection image, or a tomographic series of projection images, requiring $2 \mathrm{D}$ collimation which is "flux expensive".

Using simple geometric transformations, the above argument laid out for parallel beam geometry, i.e., plane wave illumination can be generalized to the cone beam case. In this case, a point in the sample plane at distance $z_{01}$ behind the source receives wave vectors with a width $\sigma_{i} \simeq p / z_{01}$, where $p$ is the size of the source, and the signal is recorded in the detection plane at distance $z_{12}$ behind the object. The small source size has two immediate benefits: (i) increased partial coherence required for phase contrast, and (ii) allows larger geometric magnification $M=1+z_{12} / z_{01}$ of the object without blurring effects. Note that high resolution requires small source size to minimize blurring of the image by convolution with the source size. According to the Fresnel scaling theorem [6], the wave optical treatment preserves the geometric magnification of geometrical optics, but at a scaled propagation distance (effective propagation distance) $z_{\mathrm{eff}}=z_{12} / M$.

For illustration of the 3DRT measurement and reconstruction, consider the geometry with anisotropic source as sketched in Fig. 1(a). In essence, the 3DRT requires area integrals over a set of parallel planes through the object, and yields one-dimensional (1D) curves $g_{\theta, \phi}(s)$ along the normal of the planes $s$. Normal vectors $\underline{n}_{\theta, \phi}$ have to be sampled on the unit sphere, as described by the 3DRT operating on the object function $f(x), \underline{x} \in \mathbb{R}^{3}$

$g_{\theta, \phi}(s)=(\mathcal{R} f)\left(\underline{n}_{\theta, \phi}, s\right):=\int_{\mathbb{R}^{3}} d^{3} x f(\underline{x}) \delta\left(\underline{x} \cdot \underline{n}_{\theta, \phi}-s\right)$,

where $\mathcal{R}$ denotes the operator of the Radon transform. Hence, the 3DRT is based on integrals over planes rather than integrals over lines, as the 2DRT. A simple way to obtain an area integral $g_{\theta, \phi}(s)$ from a projection image is to integrate the measured 2D image along the low-resolution direction. If the measured 1D signals $g_{\theta, \phi}(s)$ are correctly sampled for different directions of the unit vector $\underline{n}_{\theta, \phi}$, the 3D Fourier space is fully sampled, as illustrated in Fig. 1(b).

To test the feasibility of the 3DRT, we first turn to numerical simulations. For the simulation, a volume of $256^{3}$ voxels with 40 randomly distributed homogeneous spheres of 20 pixel radius, as shown in Fig. 2(a), is projected to 1D-absorption profiles $g_{\theta, \phi}(s)$ for 8001 different pairs of angles $(\theta, \phi)$. To simulate anisotropic source conditions, the $2 \mathrm{D}$ projections [cf. Fig. 2(a)] are convolved with a
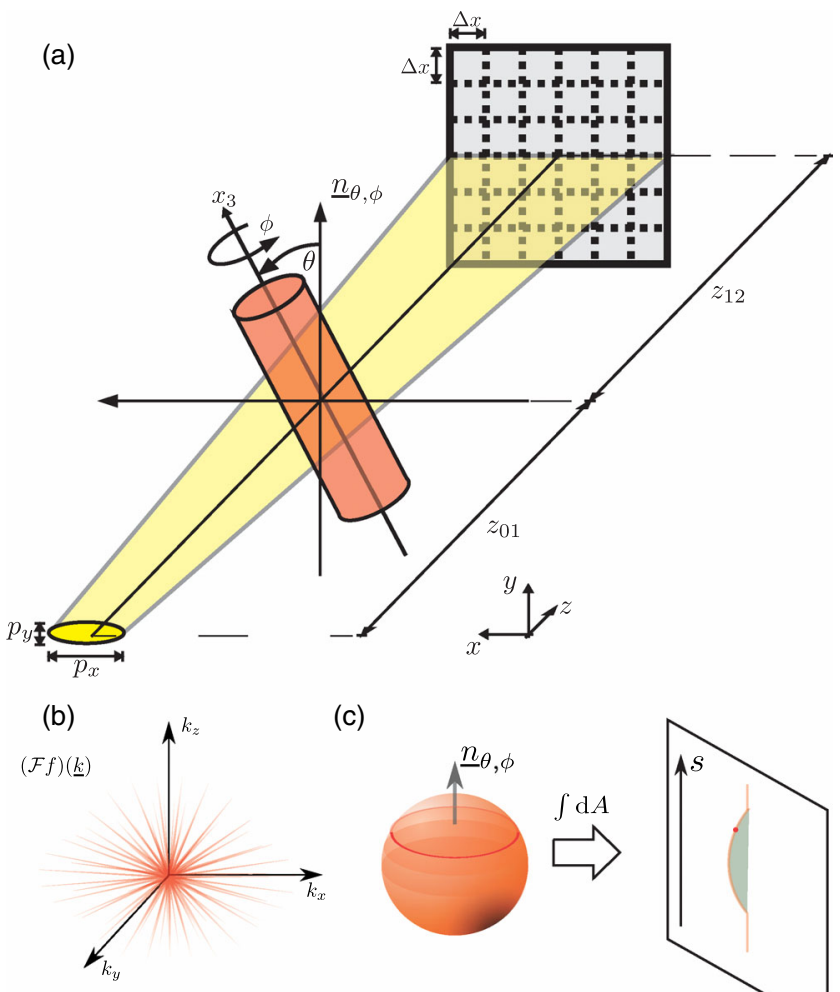

(c)

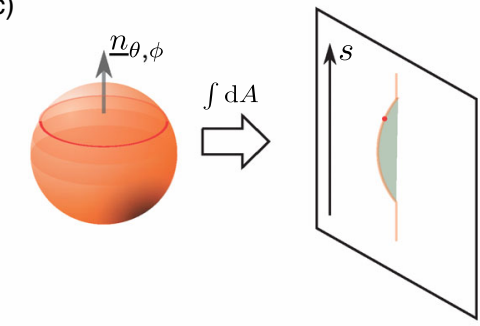

FIG. 1. Schematic of a particular realization of a generalized tomography geometry based on the 3DRT. (a) The tomographic rotation axis is successively tilted by an angle $\theta$ with respect to the laboratory $y$ axis, while the object is rotated around this axis by an angle $\phi$. 2D projection data is acquired and integrated along $x$, i.e., along the direction suffering from poor resolution and coherence. (b) Sketch of the measured signal for a 3DRT data set in 3D Fourier space resulting in a "hedgehoglike" structure. (c) Illustration of the 1D signal $g_{\theta, \phi}(s)$, corresponding to an integral over parallel planes through the object.

1D-Gaussian point spread function (PSF) with $\sigma=12 \mathrm{px}$ standard deviation along the horizontal direction as shown in Fig. 2(b). The 1D-absorption profiles are generated by summation over the horizontal direction of the projection images after convolution with the PSF. Figure 2(g) shows all 8001 absorption profiles for the pairs of angles $(\theta, \phi)$, yielding the 3DRT equivalent to the well-known sinogram in 2DRT tomography. This generalized sinogram data are the basis for the 3DRT tomographic reconstruction. To perform the 3DRT filtered back projection (FBP), the 1D-absorption profiles $g_{\theta, \phi}(s)$ are filtered by multiplication with the squared Fourier coordinate $k^{2}$ in the Fourier domain yielding the second derivative of $g_{\theta, \phi}(s)$ in real space. For details on the FBP; see Ref. [12]. To suppress noise, the $k^{2}$ filter can be combined with window functions such as Hamming or Hann windows. The volume is then reconstructed by back projection of the filtered 1D-absorption profiles. Figure 2(c) shows a reprojection of the reconstructed volume. Figures 2(d)-(f) show the power spectral densities (PSD) 

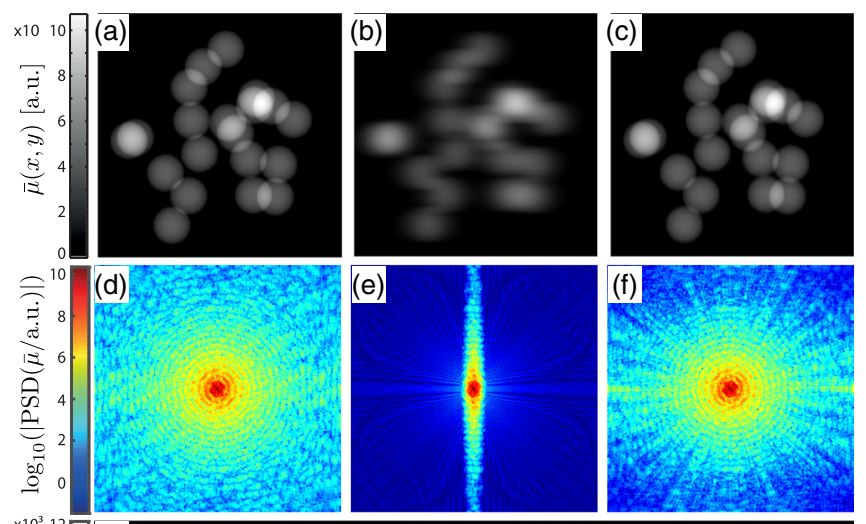

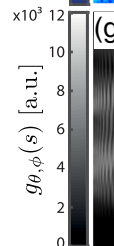

$\begin{array}{cc}\frac{80}{80} & 360 \\ \frac{8}{0} & 180 \\ \theta & \\ 0\end{array}$

$$
\begin{aligned}
& 80 \\
& \frac{0}{0} \\
& 0
\end{aligned}
$$

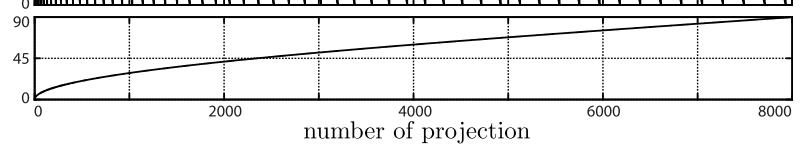

FIG. 2. Results of the simulations. (a) Projection of the original volume $\left(256^{3}\right.$ voxels $)$ with randomly distributed spheres with $20 \mathrm{px}$ radius, convolved with a 1D-Gaussian PSF of $\sigma=12 \mathrm{px}$ in the horizontal direction to simulate the blurring of anisotropic sources (b). (c) Reprojection of the reconstructed volume. (d)-(f) Power spectral densities (PSD) of the projections (a)-(c). The resolution loss in the vertical direction is clearly visible in (e), while the signal is isotropic and extends up to high Fourier components for the 3DRT FBP (f). The generalized sinogram $(\mathrm{g})$ shows the 8001 different line projections $g_{\theta, \phi}(s)$ used for the reconstruction. Each vertical line is one projection $g_{\theta, \phi}(s)$ for the pair of angles $(\theta, \phi)$ shown in the plots below. Note that the projections are arranged such that $\phi$ is scanned for each $\theta$, increasing from left to right.

corresponding to the projections shown in Figs. 2(a)-(c). The high isotropic resolution of the 3DRT is evidenced by comparing the reprojection of the reconstruction [2(c)] with the corresponding projection "raw" data [2(b)].

For a first proof of concept experiment, we chose a setup based on a sealed tube (DX-Mo10x1-P, GE-Seifert, Germany) with a molybdenum target and a pixelated detector (Timepix Hexa H05-W0154, X-ray Imaging Europe, Germany), positioned at a distance of $z_{01}+z_{12}=$ $253.7 \mathrm{~cm}$ from the source. The x-ray beam of the sealed tube with a source spot size of $1 \mathrm{~mm} \times 1 \mathrm{~mm}$ was further confined by slits with an opening of $5 \mathrm{~mm} \times 0.1 \mathrm{~mm}$ $(h \times v)$ to emulate an anisotropic source spot. A peeled hazelnut was chosen as object, positioned at $z_{01}=173 \mathrm{~cm}$ behind the source, fitting to a field of view of $450 \times 450$ detector pixels. Further details on the setup are given in Ref. [12]. The data set consists of 8001 projections $g_{\theta, \phi}(s)$,

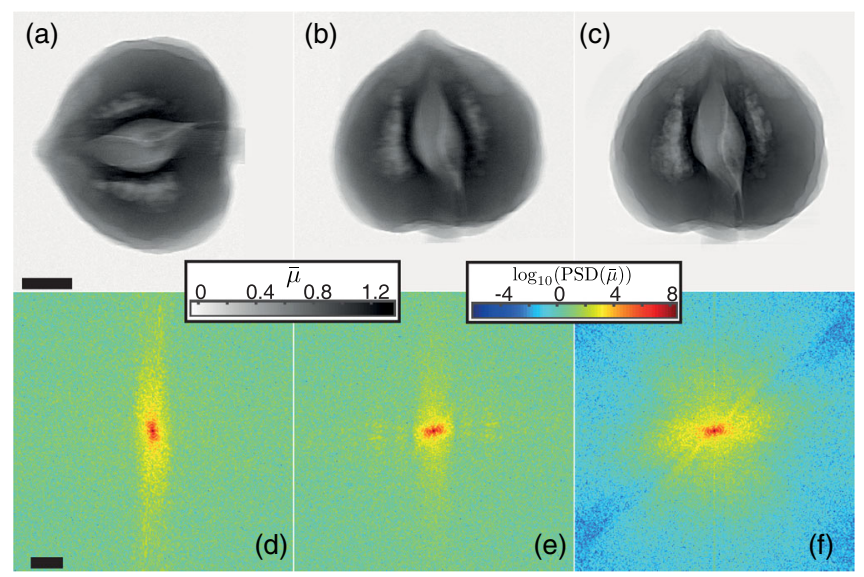

FIG. 3. Experiment: isotropic "filling" of the 3D Fourier space by the 3DRT. Projection images of the hazelnut are shown for $\theta=90^{\circ}$ (a) and $\theta=0^{\circ}$ (b), but constant $\phi$. In (a) sharp edges are transferred at the side walls of the hazelnut, corresponding to the high-resolution direction of the source, while the top and the bottom walls are blurred, while (b) shows opposite behavior. Application of the 3DRT-FBP reconstruction on the anisotropically blurred projection images yields isotropic resolution; see the reprojection of the reconstructed volume (c) [for the same $\phi$ as (a),(b)]. The PSDs (d)-(f) corresponding to (a)-(c) quantify this effect. While in (d)-(e) the signal extends over a large range in the vertical direction but decays rapidly along the horizontal direction, the signal is isotropically distributed up to high Fourier components in (f). Scale bars: $3 \mathrm{~mm}$ in (a)-(c), $3 \mathrm{~mm}^{-1}$ in (d)-(f).

chosen to evenly sample the unit sphere [20]. Figures 3(a) and 3(b) show 2D projections of the hazelnut for $\theta=90^{\circ}$ (a) and $\theta=0^{\circ}$ (b), while keeping $\phi$ constant. In Fig. 3(a) the side walls of the nut, and in 3(b) the top and bottom walls are imaged with sharp edges, corresponding to the respective high-resolution direction. Correspondingly, Fig. 3(a) shows blurred top and bottom edges, and 3(b) shows blurred side walls. A reprojection of the reconstructed volume is shown in 3(c) for the same angle $\phi$ as in 3(a) and 3(b). The reconstruction shows isotropic resolution. The PSDs [Figs. 3(d)-(f)] corresponding to the projections [Figs. 3(a)-(c)] quantify the isotropic resolution gain. Finally, Fig. 4 shows orthogonal slices through the reconstructed object for the 3DRT [4(a)] and the 2DRT [4(b)] reconstruction of the hazelnut. For the 3DRT reconstruction, from each of the 8001 anisotropically blurred projection images, 81 1D data sets were computed by evaluating the 2DRT in a sector of $\pm 10^{\circ}$ along the high resolution direction, as further detailed in Ref. [12]. The comparison clearly shows that the 3DRT provides superior image quality with high resolution in all planes.

Next, we have performed propagation based phase contrast tomography using a liquid-metal jet microfocus source (Excillum, Stockholm, Sweden) [21], at a source size of $5 \mu \mathrm{m} \times 100 \mu \mathrm{m}$, deliberately increased in one direction, while the other was kept small enough for phase contrast 


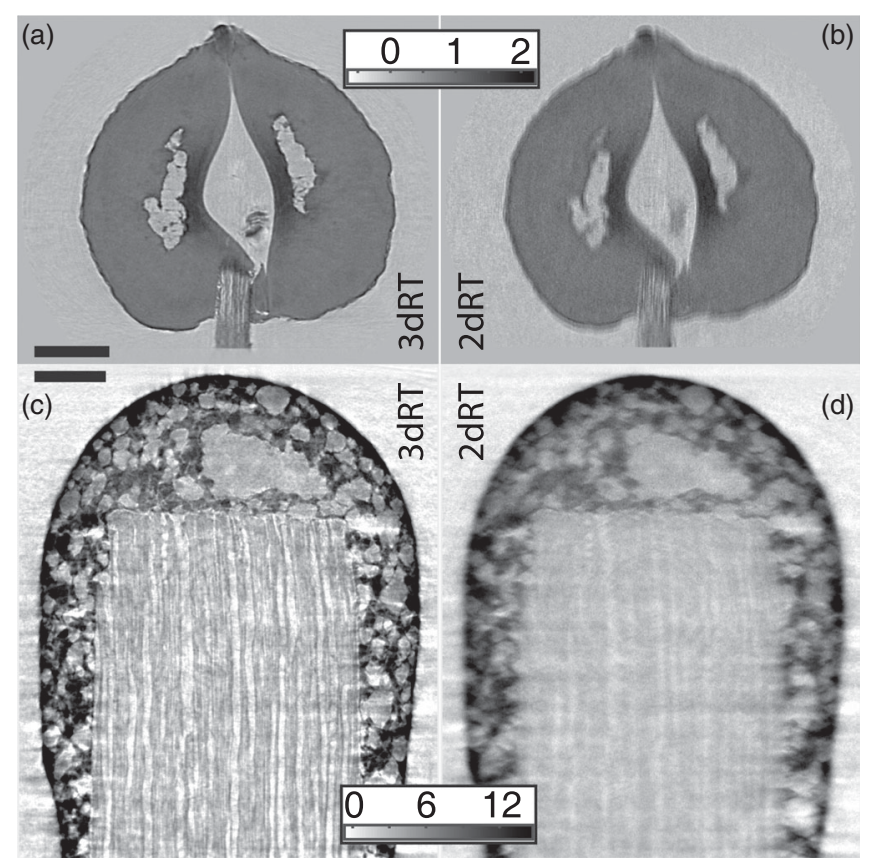

FIG. 4. Slices through the reconstructed volumes, for the incoherent experimental setting (hazelnut, top row), as well as the partially coherent setting (match head, bottom row). In both cases, the 3DRT-based tomography (left column) shows significantly better image quality than the conventional 2DRT reconstruction (right column). The 3DRT reconstruction shows even the fine details of the hazelnut in (a), and in the match head (c) sharp granular boundaries as well as the cellular wood structure. Color bars: values of $\mu$ given in $(1 / \mathrm{cm})$. Scale bars: (a),(b) $3 \mathrm{~mm}$; (c),(d) $0.5 \mathrm{~mm}$.

capability. Projection images of a match were recorded for 10011 angles in the 3DRT sampling scheme, using a fibercoupled sCMOS detector (Photonic Science, East Sussex, UK) with $6.54 \mu \mathrm{m}$ pixel size, and a field of view of $1800 \times 1800$ pixels. The spatial coherence length in the object plane for the dominating $G a K_{\alpha}$ line was about $6 \mu \mathrm{m}$ in the high coherence direction $(y)$, and thus about twice the effective object pixel size $3.1 \mu \mathrm{m}$, while it was only about $0.3 \mu \mathrm{m}$ in the low coherence direction $(x)$. Correspondingly, the phase contrast effect evidenced in the form of edge enhancement was visible along $y$ but not along $x$. An approximate phase image of the match was computed by the modified Bronnikov algorithm (MBA) [22] $\mathcal{R} \tilde{\varphi}(s, \phi, \theta) \simeq-2 \pi F \mathcal{F}^{-1}\left\{\mathcal{F}\left[\mathcal{R}\left(I_{z}(x, y) / I_{0}-1\right)\right] /\left(q_{s}^{2}+\alpha\right)\right\}$, using the Fourier filter only along the Radon coordinate $s$ instead of the conventional 2D filter kernel. Here, $F$ denotes the Fresnel number, and $\alpha$ a regularization parameter. Following Ref. [23], this approximative phase $\tilde{\varphi}(x, y)$ was then used to reconstruct the object in terms of an effective transmission function as shown in Figs. 4(c) and 4(d), with contributions from both absorption and phase interactions [12].

Figure 4 shows slices through the reconstructed volume of both experiments, i.e., the incoherent absorption CT of the hazelnut (top row), and the partially coherent CT of the match (bottom row). For both settings, the 3DRT approach (left column) shows significantly better image quality than the conventional 2DRT (right column), resulting from its compatibility with the anisotropic source sizes. It is well understood that the large bandwidth of the spectrum as well as the mixture of absorption and phase interaction impedes reconstruction of quantitative phase values. However, additional means to achieve a separation of the phase and absorption effect, such as gratings or coded apertures can be easily combined with the presented 3DRT approach. Further, we propose to operate liquid-metal-jet sources or other advanced micro-CT sources under conditions optimized for 3DRT, decreasing the source size in one direction, while extending it in the other. Using such anisotropic sources, 3DRT-based tomography should enable nanoscale resolution and phase contrast functionality in the laboratory. For the present work, the required combination of source, optics, and accuracy in the rotational positioners was not readily available and well beyond the scope of a proof-of-concept demonstration.

In summary, we have shown that the novel data recording and reconstruction scheme presented here can accommodate anisotropic sources, translating the properties of the high-resolution direction (small source size, high partial coherence) isotropically to the full 3D data set. To this end, the object has to be rotated around two axes with a continuous sampling of the unit sphere. This sampling can be obtained in different geometric ways, but differs from the dual-axis tomography which is well established in electron tomography [24]. By geometric means, one can thus escape the flux dilemma for nanoscale tomography at laboratory sources. Apart from spatial resolution, the concept helps to meet a second challenge in analytical $\mathrm{x}$-ray tomography, namely, the requirement of sufficiently high spatial coherence to achieve phase contrast.

Finally, we want to mention an entirely different motivation for using 3DRT-based tomography instead of the conventional 2DRT tomography. It can be shown mathematically, that the 3DRT has local properties in the sense that the reconstruction depends only on the local values $s$ of the Radon transformed object function $(\mathcal{R} f)(s)$ (and its derivatives), significantly facilitating local (region-ofinterest) tomography by avoiding the artifacts induced by object components outside the reconstruction volume moving in and out of projections, as commonly observed in 2DRT tomography. While the mathematics of this important difference has been pointed out before [25], it was previously believed that by the nature of the x-ray projection images, only the 2DRT case is experimentally relevant. Here we have shown by a proof-of-concept experiment, that the assumptions and geometrical conditions for area integrals can actually be reached at relevant conditions for analytical x-ray tomography.

We thank the German Research Foundation (DFG) through SFB 755/C1 for funding. 
*tsaldit@gwdg.de

[1] A. C. Kak and M. Slaney, Principles of Computerized Tomographic Imaging (IEEE, New York, 1988).

[2] T. Buzug, Computed Tomography: From Photon Statistics to Modern Cone-Beam CT (Springer-Verlag, Berlin, Heidelberg, 2008).

[3] P. Cloetens, R. Mache, M. Schlenker, and S. Lerbs-Mache, Proc. Natl. Acad. Sci. U.S.A. 103, 14626 (2006).

[4] F. Pfeiffer, C. Kottler, O. Bunk, and C. David, Phys. Rev. Lett. 98, 108105 (2007).

[5] M. Stampanoni, R. Mokso, F. Marone, J. Vila-Comamala, S. Gorelick, P. Trtik, K. Jefimovs, and C. David, Phys. Rev. B 81, 140105 (2010).

[6] D. M. Paganin, Coherent X-ray Optics (Oxford University Press, Oxford, 2006).

[7] A. Bronnikov, J. Opt. Soc. Am. A 19, 472 (2002).

[8] F. Pfeiffer, T. Weitkamp, O. Bunk, and C. David, Nat. Phys. 2, 258 (2006).

[9] K. A. Nugent, Adv. Phys. 59, 1 (2010).

[10] L. A. Feldkamp, L. C. Davis, and J. W. Kress, J. Opt. Soc. Am. A 1, 612 (1984).

[11] P. R. T. Munro, K. Ignatyev, R. D. Speller, and A. Olivio, Proc. Natl. Acad. Sci. U.S.A. 109, 13922 (2012).

[12] See Supplemental Material at http://link.aps.org/ supplemental/10.1103/PhysRevLett.116.088101 for more detailed information on methods and procedures, which includes Refs. [13-19].
[13] F. Marone and M. Stampanoni, J. Synchrotron Radiat. 19, 1029 (2012).

[14] F. Natterer, The Mathematics of Computerized Tomography (SIAM, Philadelphia, 2001).

[15] E. B. Saff and A. B. J. Kuijlaars, Math. Intell. 19, 5 (1997).

[16] A. Bronnikov, Opt. Commun. 171, 239 (1999).

[17] M. R. Teague, J. Opt. Soc. Am. 73, 1434 (1983).

[18] T. E. Gureyev, C. Raven, A. Snigirev, I. Snigireva, and S. W. Wilkins, J. Phys. D 32, 563 (1999).

[19] A. Markoe, Analytic Tomography (Cambridge University Press, Cambridge, England, 2014), p. 262.

[20] E. A. Rakhmanov, E. B. Saff, and Y. M. Zhou, Math. Res. Lett. 1, 647 (1994).

[21] U. Lundström, D. H. Larsson, A. Burvall, P. A. C Takman, L. Scott, H. Brismar, and H. M. Hertz, Phys. Med. Biol. 57, 2603 (2012).

[22] A. Groso, R. Abela, and M. Stampanoni, Opt. Express 14, 8103 (2006).

[23] Y. D. Witte, M. Boone, J. Vlassenbroeck, M. Dierick, and L. V. Hoorebeke, J. Opt. Soc. Am. A 26, 890 (2009).

[24] P. Penczek, M. Marko, K. Buttle, and J. Frank, Ultramicroscopy 60, 393 (1995).

[25] A. G. Ramm and A. I. Katsevich, The Radon Transform and Local Tomography (CRC Press, Boca Raton, 1996), p. 31 . 